\title{
SATISFACCIÓN SEXUAL FEMENINA: INFLUENCIA DE LA EDAD Y VARIEDAD DE PRÁCTICAS SEXUALES
}

\author{
Paula Iglesias Campos \\ pauicam@alumni.uv.es \\ Vicente Morell-Mengual \\ Lorena Caballero-Gascón \\ Roberta Ceccato \\ María Dolores Gil-Llario
}

Salusex-Unisexsida. Departamento de Psicología Evolutiva y de la Educación.

Universitat de València. Estudi General.

Fecha de Recepción: 2 Abril 2018

Fecha de Admisión: 10 Abril 2018

\begin{abstract}
RESUMEN
La satisfacción sexual es la respuesta afectiva y emocional surgida de la evaluación de las interacciones sexuales personales y de la percepción de la consumación del deseo erótico. La satisfacción marital, definida como la actitud hacia la interacción marital y los aspectos emocionales y estructurales de la pareja, es considerada uno de los más fuertes predictores de la satisfacción sexual. Además, existen otras variables que pueden estar interviniendo en la satisfacción sexual y marital de las mujeres. Por tanto, el objetivo de este trabajo es analizar la influencia de la edad y la variedad de prácticas sexuales y afectivas en la satisfacción sexual y marital de mujeres con pareja estable. La muestra está formada por 600 mujeres de edades comprendidas entre los 18 y 60 años $(\mathrm{M}=31.57 ; \mathrm{DT}=9.14)$ que, independientemente de su orientación sexual, mantienen una relación de pareja estable de una duración igual o superior a dos años. A todas ellas se les aplicó la Escala de Satisfacción Sexual, la Escala de Satisfacción Marital y un inventario de prácticas sexuales. Los resultados muestran una relación inversa con la edad, de manera que el grupo de menor edad presenta puntuaciones significativamente superiores en el Factor "Comunicación" de la Escala de Satisfacción Sexual, así como en la Escala de Satisfacción Marital. Además, se ha encontrado que a mayor variedad de prácticas sexuales y afectivas, tanto durante como después de la relación sexual, mayor satisfacción sexual. Por último, la satisfacción marital se relaciona de forma directa con la satisfacción sexual. Las mujeres jóvenes y con amplia variedad de prácticas sexuales y afectivas presentan una mayor satisfacción sexual y marital. Se ha evidenciado la necesidad de elaborar modelos multicausales sobre las variables implicadas en la satisfacción sexual femenina debido a la complejidad que entraña.
\end{abstract}




\section{SATISFACCIÓN SEXUAL FEMENINA: INFLUENCIA DE LA EDAD Y VARIEDAD DE PRÁCTICAS SEXUALES}

Palabras clave: satisfacción sexual; satisfacción marital; prácticas sexuales; prácticas afectivas; edad

\section{ABSTRACT}

Female sexual satisfaction: influence of age and variety of sexual practices.

Sexual satisfaction is the affective and emotional response arising from the evaluation of personal sexual interactions and the perception of the consummation of erotic desire. Marital satisfaction, defined as the attitude towards marital interaction and the emotional and structural aspects of the couple, is considered one of the strongest predictors of sexual satisfaction. There are other variables that may intervene in the sexual and marital satisfaction of women. The objective of this study is to analyze the influence of age, and the variety of sexual and affective practices, on sexual and marital satisfaction of women with a stable partner. The sample consists of 600 women between the ages of 18 and $60(M=31.57 ; S D=9.14)$ who, regardless of their sexual orientation, maintain a stable relationship with a duration of two or more years. The Sexual Satisfaction Scale, the Marital Satisfaction Scale and a sexual practices inventory were applied to all of them. An inverse relationship with age is observed, with the younger age group presenting significantly higher scores in the "Communication" Factor of the Sexual Satisfaction Scale, as well as in the Marital Satisfaction Scale. It was also has been found that a greater variety of sexual and affective practices, both during and after sexual intercourse, is related to a greater sexual satisfaction. Finally, marital satisfaction is directly related to sexual satisfaction. Young women with a wide variety of sexual and affective practices have greater sexual and marital satisfaction. The complexity of the study suggests the need for multicausal models on the variable involved in female sexual satisfaction.

Keywords: sexual satisfaction; marital satisfaction; sexual practices; affective practices; age

\section{ANTECEDENTES}

La satisfacción sexual es la respuesta afectiva y emocional surgida de la evaluación de las interacciones sexuales personales y de la percepción de la consumación del deseo erótico. Puede ser definida, por tanto, como el nivel de agrado, bienestar y ajuste presentado frente a una interacción sexual, que se evidencia a través de las reacciones emocionales experimentadas y la comunicación verbal y no verbal con la pareja (Jiménez, 2010).

Sin embargo, a pesar de la estrecha relación que este complejo constructo guarda con el estado de salud y la calidad de vida (Carrobles, Gámez-Guadix y Almendros, 2011), son escasos los estudios acerca de la satisfacción sexual en las relaciones a largo plazo. Debido a la tradicional concepción de la sexualidad como una función relacionada con la reproducción, y no como una función placentera en sí misma, es muy reciente el interés de la investigación acerca de la relación entre la sexualidad y la calidad de vida, considerando en particular las variables implicadas en la satisfacción sexual de mujeres con pareja estable (Muise, Giang y Impett, 2014).

En este sentido, se ha constatado que la calidad de las relaciones sexuales disminuye lentamente con el tiempo (Mark y Herbenick, 2014), debido a que el deseo sexual es típicamente más alto al principio de una relación, con caídas a medida que aumenta la duración de la misma (Murray y Milhausen, 2012). Por ello, para definir la relación a largo plazo, seguiremos la delimitación propuesta por Hazan y Zeifman (1994), quienes consideran que el apego ya se ha constituido a partir de los dos primeros años de relación, considerándose en ese momento como relación de pareja estable.

La literatura existente en este ámbito señala distintas relaciones entre la satisfacción sexual y aspectos demográficos, como la edad, y aspectos conductuales, como las prácticas sexuales. 
En cuanto a la edad, se ha encontrado que la satisfacción sexual disminuye a medida que ésta aumenta (Christopher y Sprecher, 2000; Young, Denny, Young y Luquis, 2000), relacionándose una mayor edad con actitudes sexuales más negativas, menos deseo sexual y menos actividad sexual (Katz y Marshall, 2003; Mark y Herbenick, 2014). Por otro lado, Masters y Johnson (1966) señalaron que muchas de las parejas mayores que estudiaron continuaban disfrutando del sexo, siempre y cuando no hubieran dejado de tenerlo (Schwartz y Young, 2009), por lo que la práctica continuada podría interactuar con la variable edad.

Poco se ha estudiado sobre el papel que tiene la variedad de prácticas sexuales. Algunas investigaciones sugieren que las parejas que tienen una mayor frecuencia de afecto, una mayor duración de los juegos previos (Muise, Giang y Impett, 2014) y que participan en una mayor variedad de comportamientos y prácticas sexuales (Gossman et al., 2003; Scwart y Young, 2009), presentan mayor satisfacción y cuentan con menos problemas sexuales, especialmente a edades avanzadas (Hinchliff y Gott, 2008; Muise, Giang y Impett, 2014; Potts, Grace, Vares y Gavey, 2006).

Del mismo modo, no es menor la importancia de las conductas afectivas al final de la práctica sexual, considerado como un momento crítico para reforzar la unión y la intimidad de la pareja (Hughes y Kruger, 2011; Muise, Giang y Impett, 2014). La duración y la calidad del afecto en este momento se asocia con una mayor satisfacción sexual y marital, especialmente en las mujeres, quienes valoran más el afecto después de la relación sexual y suelen reportar menos satisfacción cuando su cantidad deseada no se ve satisfecha (Muise, Giang y Impett, 2014).

Por otro lado, la satisfacción marital, definida como la actitud hacia la interacción marital y los aspectos emocionales y estructurales de la pareja (Flores, Roldan y Flores, 2012), es considerada uno de los más fuertes predictores de la satisfacción sexual, tal y como ha sido demostrado a lo largo de muchos estudios (Mark y Herbenick, 2014; Milhausen, Buchholz, Opperman y Benson, 2015; Sánchez Fuentes, 2016), en los que se concluye que las personas con una mayor satisfacción de la relación tienden a reportar una mayor satisfacción sexual (Byers, 2005; Milhausen, Buchholz, Opperman y Benson, 2015; Sprecher, 2002).

Por último, los estudios sobre las contribuciones no sexuales a la satisfacción sexual hablan, a menudo, de la comunicación como un factor clave (Purnine y Carey, 1997). Autores como Byers (2005) y Haavio-Manila y Kontula (1997) consideran que las parejas que mantienen comunicación íntima de alta calidad, tienen mayor satisfacción sexual y marital que aquellas con baja comunicación.

\section{OBJETIVO}

Por tanto, el objetivo del presente trabajo es estudiar las variables implicadas en la satisfacción sexual de un grupo de mujeres con pareja estable. En concreto, este estudio analiza la relación entre la edad, la satisfacción marital, la variedad de prácticas sexuales y la satisfacción sexual en este grupo.

\section{PARTICIPANTES}

La muestra está formada por 600 mujeres, de edades comprendidas entre los 18 y 60 años (M = 31.57; DT = 9.14), que, independientemente de su orientación sexual, cumplían los siguientes criterios de inclusión: mujeres con pareja estable de duración igual o superior a dos años y con actividad sexual dentro de la misma.

El $75.3 \%$ de las participantes se define como heterosexual, el $19.6 \%$ como bisexual y el $5.1 \%$ como homosexual. Con respecto al nivel educativo, el $78.4 \%$ posee estudios universitarios o postuniversitarios, el $19.3 \%$ estudios secundarios y el $2.3 \%$ no tiene estudios o son primarios. 


\section{SATISFACCIÓN SEXUAL FEMENINA: INFLUENCIA DE LA EDAD Y VARIEDAD DE PRÁCTICAS SEXUALES}

\section{MÉTODO}

\section{Instrumentos}

Escala de Satisfacción Sexual (Jiménez, 2010). Está formada por 33 ítems con formato de respuesta tipo Likert de cinco puntos y ofrece información sobre dos factores: Comunicación y Reacción Emocional. La Comunicación hace referencia al proceso de interacción de la pareja y a sus estilos de interacción verbal y no verbal. Por otra parte, la Reacción Emocional corresponde a las emociones y sentimientos que expresa la persona en relación a sus relaciones sexuales: antes, durante 0 después de las mismas. En el estudio original se obtuvo un Alfa de Cronbach total de la escala de 0.92, y en nuestro estudio se ha obtenido un coeficiente de fiabilidad de 0.90 .

Escala de Satisfacción Marital (Pick y Andrade, 1988) formada por 24 afirmaciones distribuidas en tres subescalas: Satisfacción con la interacción conyugal, Satisfacción con los aspectos emocionales del cónyuge y Satisfacción con los aspectos organizacionales y estructurales de la relación. En nuestro estudio se ha obtenido un Alfa de Cronbach de 0.90 para el total de la escala.

Inventario de prácticas sexuales y aspectos afectivos, elaborado ad hoc y formado por 14 ítems que evalúan la variedad de prácticas sexuales y los comportamientos afectivos tras la interacción sexual.

\section{Procedimiento}

La batería de evaluación fue difundida a través de redes sociales y, especialmente, en páginas dedicadas a la sexualidad femenina. Todas las participantes lo hicieron de forma voluntaria y anónima. Antes de dar su consentimiento se les informaba de la confidencialidad y privacidad de los datos extraídos, así como de la imposibilidad de identificación a través de sus respuestas, y se les indicaba explícitamente que la información extraída del mismo sería utilizada única y exclusivamente con fines de investigación.

El tiempo medio requerido para cumplimentar el cuestionario era de, aproximadamente, quince minutos. Seguidamente, los datos fueron analizados con el software estadístico SPSS versión 24.

\section{RESULTADOS}

La satisfacción sexual presenta una relación inversa con la edad $\left(r=-.090^{\star}\right)$, de manera que dicha satisfacción es mayor en edad inferiores. En la tabla 1 podemos observar que el grupo de menor edad presenta puntuaciones significativamente más elevadas que el grupo de edad superior en el primer Factor de Satisfacción Sexual: Comunicación $\left(F=5.487^{*}\right)$. 
Tabla 1. Comparación de medias de satisfacción sexual en diferentes edades.

\begin{tabular}{|c|c|c|c|c|c|}
\hline & $\begin{array}{c}(1) \\
18-30 \\
\bar{X} \\
(D T)\end{array}$ & $\begin{array}{c}(2) \\
31-45 \\
\bar{X} \\
(D T)\end{array}$ & $\begin{array}{c}(3) \\
46-60 \\
\bar{X} \\
(D T)\end{array}$ & $\mathbf{F}$ & Post hoc \\
\hline SaSex1 & $\begin{array}{c}62.64 \\
(9.041)\end{array}$ & $\begin{array}{c}61.15 \\
(9.348)\end{array}$ & $\begin{array}{c}58.82 \\
(9.992)\end{array}$ & $5.487^{*}$ & $1>3$ \\
\hline SaSex 2 & $\begin{array}{c}69.95 \\
(5.950)\end{array}$ & $\begin{array}{c}69.56 \\
(6.384)\end{array}$ & $\begin{array}{c}70.72 \\
(6.122)\end{array}$ & .945 & \\
\hline SaSexTot & $\begin{array}{c}132.59 \\
(12.849)\end{array}$ & $\begin{array}{c}130.71 \\
(14.164)\end{array}$ & $\begin{array}{r}129.54 \\
(12.453)\end{array}$ & 2.299 & \\
\hline
\end{tabular}

Nota: SaSex1: Satisfacción sexual: Factor 1: Comunicación; SaSex2: Satisfacción sexual: Factor 2: Reacción Emocional; SaSexTot: Satisfacción Sexual Total.

Tabla 2. Correlaciones entre las variables: satisfacción sexual, variedad de prácticas sexuales, comportamientos afectivos y satisfacción marital.

\begin{tabular}{ccccccc}
\hline & SaSex1 & SaSex2 & SaSexTot & PractSex & Afect & SaMarit \\
\hline SaSex1 & 1 & & & & & \\
SaSex2 & $.462 * *$ & 1 & & & & \\
SaSexTot & $.315 * *$ & $.307 * *$ & 1 & & & \\
PractSex & $.224 * *$ & $.142 * *$ & $.222^{* *}$ & 1 & .12 & \\
Afec & $.270^{* *}$ & $.221 * *$ & $.177^{* *}$ & .102 & 1 \\
SaMarit & $.351 * *$ & $.339 *$ & $.402 * *$ & $.121 * *$ & .143 & 1
\end{tabular}

Nota: SaSex1: Satisfacción sexual: Factor 1: Comunicación; SaSex2: Satisfacción sexual: Factor 2: Reacción Emocional; SaSexTot: Satisfacción Sexual Total; PractSex: Variedad de Prácticas Sexuales; Afec: Comportamientos afectivos; SaMarit: Satisfacción Marital.

En la tabla 2 podemos observar como la satisfacción sexual correlaciona de forma directa y significativa con la variedad de prácticas durante la relación sexual $\left(r=.222^{\star *}\right)$, así como con los comportamientos afectivos después del sexo $\left(r=.177^{\star *}\right)$. Por lo que, a mayor variedad de prácticas 


\section{SATISFACCIÓN SEXUAL FEMENINA: INFLUENCIA DE LA EDAD Y VARIEDAD DE PRÁCTICAS SEXUALES}

sexuales y afectivas, tanto durante como después de la relación sexual, mayor es la satisfacción sexual.

Y lo mismo ocurre con la satisfacción marital, que se relaciona de forma directa con los dos factores que componen la Escala de Satisfacción Sexual: Comunicación $\left(r=.351^{* *}\right)$ y Reacción Emocional $\left(r=.339^{\star}\right)$, así como con la puntuación total de la escala $\left(r=.402^{\star *}\right)$. Por tanto, a mayores puntuaciones de satisfacción marital, también se darán unas altas puntuaciones en satisfacción sexual, una mayor comunicación sexual en la pareja y una mayor satisfacción en cuanto a las emociones y sentimientos expresados en la relación con respecto a las interacciones sexuales.

\section{DISCUSIÓN Y CONCLUSIONES}

A partir de los hallazgos encontrados y en relación con trabajos previos, la presente investigación muestra la relación que presentan la edad, la satisfacción marital, la variedad de prácticas sexuales y la calidad de la relación afectiva con la satisfacción sexual de las mujeres con pareja estable. En relación con la edad, los datos resultantes apoyan que la satisfacción sexual de las mujeres disminuye con la edad, tal y como anteriormente habían demostrado estudios como los de Christopher y Sprecher (2000), Katz y Marshall (2003), Mark y Herbenick (2014) y Young, Denny, Young y Luquis (2000).

Por otro lado, nuestros resultados apoyan que la variedad de prácticas sexuales y la expresión de afecto, tanto durante la relación sexual, tal y como concluían Gossman et al. (2003) y Schwart y Young (2009), como inmediatamente después de mantenerla, en la línea de los resultados obtenidos por Hughes y Kruger (2011), se relaciona con una mayor satisfacción sexual.

Por último, la satisfacción marital o de pareja es una de las variables que más se relaciona con la satisfacción sexual, habiéndose mostrado esta relación en nuestros resultados, así como en otros estudios recientes como los de Mark y Herbenick (2014), Milhausen, Buchholz, Opperman y Benson (2015) y Sánchez Fuentes (2016).

La comunicación ha destacado en los análisis realizados como un factor clave en las relaciones de pareja, en la línea de lo obtenido por Purnine y Carey (1997), asociándose a las mujeres más jóvenes, con buena relación de pareja y con amplia variedad de prácticas sexuales y adecuadas manifestaciones afectivas, que son quienes presentan una mayor satisfacción sexual. Estos resultados concuerdan con los obtenidos por Haavio-Manila y Kontula (1997) y Byers (2005), quienes mantienen que las parejas que presentan una comunicación de alta calidad sobre el sexo tienen mayor satisfacción que aquellas parejas con baja comunicación.

Debemos tener precaución a la hora de generalizar los resultados obtenidos pues, tal y como han demostrado investigaciones anteriores, las personas que acceden voluntariamente a participar en estudios relacionados con la sexualidad tienden a ser sustancialmente más experimentadas sexualmente y tienen actitudes sexuales más liberales que aquellas que no lo hacen, siendo más positivas hacia todos los aspectos relacionados con el sexo (Boynton, 2003; Fallis, Rehman y Purdon, 2014).

Todos estos hallazgos ponen de manifiesto la necesidad de elaborar modelos multicausales sobre las variables implicadas en la satisfacción sexual de las mujeres debido a la complejidad que entraña.

\section{REFERENCIAS}

Boynton, P. M. (2003). I'm just a girl who can't say no?: Women, consent, and sex research. Journal of Sex \& Marital Therapy, 29(Suppl. 1), 23-32.

Byers, E. S. (2005). Relationship satisfaction and sexual satisfaction: A longitudinal study of individuals in long-term relationships. Journal of Sex Research, 42(2), 113- 118. 
Carrobles, J. A., Gámez-Guadix, M., y Almendros, C. (2011). Funcionamiento sexual, satisfacción sexual y bienestar psicológico y subjetivo en una muestra de mujeres españolas. Anales De Psicología, 27(1), 27-34.

Christopher, F. S., y Sprecher, S. (2000). Sexuality in marriage, dating, and other relationships: A decade review. Journal of Marriage and Family, 62(4), 999- 1017.

Fallis, E. E., Rehman, U. S., y Purdon, C. (2014). Perceptions of partner sexual satisfaction in heterosexual committed relationships.Archives of Sexual Behavior, 43(3), 541-550.

Flores, S. B., Roldan, W., y Flores, W. (2012). Factores de enriquecimiento, mantenimiento, comunicación y bienestar en la relación marital. Revista De Psicología GEPU, 3(1), 37-62.

Gossmann, I., Mathieu, M., Julien, D., y Chartrand, E. (2003). Determinates of sex initiation frequencies and sexual satisfaction in long-term couples' relationships. The Canadian Journal of Human Sexuality, 12, 169-181.

Haavio-Manilla, E. y Kontula, O. (1997). Correlates of increased sexual satisfaction. Archives of Sexual Behavior, 26(4), 399-418.

Hazan, C. y Zeifman, D. (1994). Sex and the psychological tether. En D. Perlman y K. Bartholomew (Eds.), Advances in personal relationships (Vol. 5, pp. 151-180). London: Kingsley.

Hinchliff, S.,y Gott,M. (2008).Challenging social myths and stereotypes of women and aging: Heterosexual women talk about sex. Journal of Women and Aging, 20, 65- 80.

Hughes, S. M., y Kruger, D. J. (2011). Sex differences in post-coital behaviors in long- and-shortterm mating: An evolutionary perspective. Journal of Sex Research, 48(5), 496-505.

Jiménez, 0. R. R. (2010). Relación entre satisfacción sexual, ansiedad y prácticas sexuales. Pensamiento Psicológico, 7(14).

Katz, S., y Marshall, B. (2003). New sex for old: Lifestyle, consumerism, and the ethics of aging well. Journal of Aging Studies, 17(1), 3-16.

Mark, K. P. (2014). The impact of daily sexual desire and daily sexual desire discrepancy on the quality of the sexual experience in couples. The Canadian Journal of Human Sexuality, 23(1), 27-33.

Mark, K. P., y Herbenick, D. (2014). The influence of attraction to partner on heterosexual women's sexual and relationship satisfaction in long-term relationships. Archives of Sexual Behavior, 43(3), 563-570.

Masters, W. H., y Johnson, V. E. (1966). Human sexual response. Boston, Little, Brown \& Co, 238240.

Milhausen, R. R., Buchholz, A. C., Opperman, E. A., y Benson, L. E. (2015). Relationships between body image, body composition, sexual functioning, and sexual satisfaction among heterosexual young adults. Archives of Sexual Behavior, 44(6), 1621-1633.

Muise, A., Giang, E., y Impett, E. A. (2014). Post sex affectionate exchanges promote sexual and relationship satisfaction. Archives of Sexual Behavior, 43(7), 1391- 1402.

Murray, S.H., y Milhausen, R.R. (2012). Sexual desire and relationship duration in young men and women. Journal of Sex \& Marital Therapy, 38(1), 28-40.

Pick, S. y Andrade, P. (1988). Desarrollo y validación de la Escala de Satisfacción Marital. Psiquiatría, 4(1), 9-20.

Potts, A., Grace, M., Vares, T., y Gavey, N. (2006). 'Sex for life'? Men's counter-stories on 'erectile dysfunction', male sexuality and ageing. Sociology of Health \& Illness, 28(3), 306-329.

Purnine, D. M., y Carey, M. P. (1997). Interpersonal communication and sexual adjustment: The roles of understanding and agreement. Journal of Consulting and Clinical Psychology, 65(6), 1017-1025.

Sánchez Fuentes, M. M. (2016). Satisfacción sexual: Análisis de factores asociados e implicaciones 


\section{SATISFACCIÓN SEXUAL FEMENINA: INFLUENCIA DE LA EDAD Y VARIEDAD DE PRÁCTICAS SEXUALES}

clínicas. (Tesis doctoral). Universidad de Granada.

Schwartz, P., y Young, L. (2009). Sexual satisfaction in committed relationships. Sexuality Research \& Social Policy, 6(1), 1-17.

Sprecher, S. (2002). Sexual satisfaction in premarital relationships: Associations with satisfaction, love, commitment, and stability. Journal of Sex Research, 39(3), 190-196.

Young, M., Denny, G., Young, T., y Luquis, R. (2000). Sexual satisfaction among married women. American Journal of Health Studies, 16(2), 73. 